\title{
ON STABILITY AND UNIQUENESS OF FLUID FLOW THROUGH A RIGID POROUS MEDIUM*
}

\author{
BY \\ K. A. PERICAK-SPECTOR \\ Southern Illinois University-Carbondale and Institute for Mathematics and Its Applications, Minneapolis
}

\begin{abstract}
We study a set of equations describing the flow of an incompressible viscous fluid through a rigid porous medium. Existence, uniqueness and stability results are established for the case of a region impregnated with fluid, and uniqueness for an unsaturated region.
\end{abstract}

I. Introduction. In this paper we study a set of equations which have been proposed by Williams [1] to describe the flow of an incompressible, viscous fluid through a rigid porous medium. ${ }^{1}$

In Sec. II we discuss the equations in detail, examining two basic situations: fluid flowing in a saturated region (all the pores are filled with fluid) and fluid flowing in an unsaturated region (here it is possible to have air present in the pores). We go on to show how the equations are related to the classical laws of Darcy and Fick and derive generalizations of these laws by reintroducing some of the neglected terms.

In Sec. III the question of uniqueness in an unsaturated region is discussed. It will be shown that even with zero viscosity uniqueness can still occur in some particular cases.

In Sec. IV we consider uniqueness in the case of a saturated body. Three situations will be considered, namely an inhomogeneous and time-dependent region will be studied first. Secondly, the medium is assumed to be inhomogeneous and time-independent. Finally, a homogeneous and time-independent body is studied. In the last case the equations in question resemble those of the incompressible Navier-Stokes with a dissipative term added on. It will be shown that uniqueness may still occur even at a high Reynolds number, if the dissipative term is sufficiently large.

\footnotetext{
* Received July 9, 1982. The author wishes to thank Professor W. O. Williams for suggesting this problem and for many discussions. This work was partially supported by the National Science Foundation and the Institute for Mathematics and Its Applications at the University of Minnesota.

${ }^{1}$ The equations in [1] were obtained by specialization from a set of equations first suggested by Williams [2] and Sampaio and Williams [3]. In fact, the equations given by [2] and [3] differ from those in [1] in the form of the indeterminant pressure term (cf. Pericak-Spector and Williams [4]). The equations herein are a specialization of equations derived by Aifantis [5] and Nunziato and Walsh [6]. Cf. Williams [1], Aifantis [5], Nunziato and Walsh [6], Drumheller [7], Bedford and Drumheller [8] and Kenyon [9] for a more detailed discussion of models describing fluid flow through a porous medium.
} 
Finally, in Sec. V we discuss stability in a saturated, homogeneous and time-independent region. Now it will be shown that stability may occur even at a high Reynolds number again as long as the dissipative term is sufficiently large. We also observe that the standard theorems of existence and uniqueness of weak solutions still hold in this generalized case.

\section{Preliminaries.}

A. Notation. We use the standard inner product $\mathbf{u} \cdot \mathbf{v}$ on $\mathbf{R}^{3}$ while on

$$
\operatorname{Lin}=\text { the space of all tensors }
$$

we use the inner product

$$
A \cdot B=\operatorname{tr}\left(A B^{T}\right)
$$

with $B^{T}$ the transpose of $B$ and tr the trace. All norms are denoted by $\|\cdot\| .1$ denotes the identity tensor.

The tensor product of two vectors $\mathbf{u}$ and $\mathbf{v}$, denoted by $\mathbf{u} \otimes \mathbf{v}$ is a tensor with components $(\mathbf{u} \otimes \mathbf{v})_{i j}=u_{i} v_{j}$.

We write $\nabla$ and div for the gradient and divergence operators in $\mathbf{R}^{3}$ : for a vector field $\mathbf{u}, \nabla \mathbf{u}$ is the tensor field with components $(\nabla \mathbf{u})_{i j}=\partial u_{i} / \partial x_{j}$; for a tensor field $S$, $\operatorname{div} S$ is the vector field with components $\sum_{j=1}^{3} \partial S_{i j} / \partial x_{j}$. We write the symmetric part of $\nabla \mathbf{u}$ as $\widehat{\nabla \mathbf{u}}$ :

$$
\widehat{\nabla \mathbf{u}}=\frac{1}{2}\left(\nabla \mathbf{u}+\nabla \mathbf{u}^{T}\right) .
$$

Finally, we let $\mathbf{u}_{t}$ denote the time derivative of $\mathbf{u}$.

B. Formulation of equations. Our equations are based on the formulation of mixture theory due to Gurtin and de la Penha [10], Oliver [11], Williams [2] and Sampaio and Williams [3]. Taking the continuum point of view we consider a fluid body $\Re_{f}$ moving in a rigid porous body $\mathscr{B}_{b}$. Interactions between the fluid and solid are due to their coincidence in space. The balance of mass and force equations, specialized from [1], are

$$
\begin{gathered}
\rho \phi \mathbf{u}_{t}+\rho \phi \nabla \mathbf{u u}=-\nabla p+\operatorname{div}(\eta(\phi) \widehat{\nabla \mathbf{u}})-\alpha(\phi) \mathbf{u}-\nabla \beta(\phi), \\
\phi_{t}+\operatorname{div}(\phi \mathbf{u})=0 .
\end{gathered}
$$

Here $\rho$ is the actual density of the incompressible fluid (and is constant), $\phi$ is the volume fraction (volume of fluid per total volume), $p$ is the pressure and $\mathbf{u}$ the velocity. $\eta$ denotes the viscosity, $\alpha$ the drag and $\beta$ the capillary coefficient of the fluid. The last three terms are scaler-valued functions of the volume fraction $\phi$. Notice there is no equation for the balance of mass or force for the solid, because we have assumed the solid to be rigid and motionless.

We now suppose the medium occupies a region, $\mathscr{B}$, with boundary $\partial \mathscr{B}$. We will let $\mathbf{n}$ denote the exterior normal to the boundary.

There are two basic situations to be considered. The first is when a region of the solid is completely filled with fluid $\left[\phi(\mathbf{x}, t) \equiv \phi_{0}\right]$. This is said to be a saturated region. Looking at (2.1) we see that the capillary term and the rate of change of the volume flux are zero so that the equations will reduce to

$$
\begin{gathered}
\rho \phi_{0} \mathbf{u}_{t}+\rho \phi_{0} \nabla \mathbf{u u}=-\nabla p+\eta_{0} \Delta \mathbf{u}-\alpha_{0} \mathbf{u}, \\
\operatorname{div} \mathbf{u}=0,
\end{gathered}
$$


where $\eta_{0}=\frac{1}{2} \eta\left(\phi_{0}\right)$ and $\alpha_{0}=\alpha\left(\phi_{0}\right)$. Notice the similarity of these equations to the incompressible Navier-Stokes equations, the only addition being the drag term. We shall show that stability and uniqueness holds for a larger class of initial data than in the Navier Stokes equations and that, in particular, it is possible to have a well-behaved equation (in the classical sense) with a nonviscous fluid. Existence, uniqueness and stability results (in the weak sense) valid for the Navier-Stokes system will be shown to hold.

In the second case we consider a region in which the pores are not filled with fluid. We will call this an unsaturated region. It is shown by Pericak-Spector and Williams [4], that the pressure gradient is then zero. Physically one can argue that a uniform pressure is maintained, in the unsaturated case, by the neglected third constituent, e.g., air, that occupies the remainder of the volume. Thus, Eqs. (2.1) become

$$
\begin{gathered}
\rho \phi \mathbf{u}_{t}+\rho \phi \nabla \mathbf{u u}= \\
\operatorname{div}(\eta(\phi) \widehat{\nabla \mathbf{u}})-\alpha(\phi) \mathbf{u}-\nabla \beta(\phi), \\
\phi_{t}+\operatorname{div}(\phi \mathbf{u})=0 .
\end{gathered}
$$

Equations (2.3) are similar to the equations of a compressible viscous fluid, again with the drag term $-\alpha(\phi) \mathbf{u}$ added and $\beta(\phi)$ replacing the pressure term. ${ }^{2}$

We will now show that under certain restrictions equations (2.1) reduce to the classical laws of Darcy and Fick. Then, relaxing those restrictions we will produce several "corrected" versions of these laws.

First we consider a saturated region in which steady state conditions prevail. If the cross section is large, so that viscous effects are small, and inertia can be neglected, so that the only driving force is an applied pressure gradient, then the,momentum equation reduces to $\nabla p=-\alpha_{0} u$. This is Darcy's Law. To form a "correction" to this equation we assume only that the flow is saturated; the momentum equation is

$$
-\nabla p-\rho \phi_{0} \mathbf{u}_{t}-\rho \phi_{0} \nabla \mathbf{u u}+\eta_{0} \Delta \mathbf{u}-\alpha_{0} \mathbf{u}=0 .
$$

Ignoring inertia, this equation was first studied by Brinkham [13, 14]; in [15], Brinkman extended his derivation to include inertia and arrived at (2.4). ${ }^{3}$

To derive Fick's law let us consider an unsaturated region. As before, we will neglect viscosity and inertial. Assuming $\beta$ is $C^{2}$ we see that the momentum equation is just $\alpha \mathbf{u}=-\beta^{\prime} \nabla \phi$, where the prime denotes the derivative with respect to $\phi$. Substituting this into $(2.3)_{2}$ yields the standard diffusion equation

$$
\phi_{t}-\operatorname{div}(D \nabla \phi)=0,
$$

with coefficient of diffusion $D=\beta^{\prime} \phi / \alpha$.

We next consider an unsaturated region and assume $\eta$ and $\beta$ are $C^{2}$ to derive a "corrected" diffusion equation which includes viscous and inertial effects. Multiplying

\footnotetext{
${ }^{2}$ For a one dimensional inviscid model, Pericak-Spector [12] considered the question of existence of global smooth solutions.

${ }^{3}$ For further references see Bird, Steward, and Lightfoot [16] and Scheidegger [17]. For a more detailed discussion of (2.4) see Aifantis [5].
} 
equation (2.3), by $\phi / \alpha$ and taking the divergence of the result we arrive at

$$
\begin{aligned}
\rho \frac{\phi^{2}}{\alpha} \operatorname{div} \mathbf{u}_{t}= & \frac{1}{\alpha} \eta \phi \operatorname{div} \Delta \mathbf{u}-\operatorname{div}(\phi \mathbf{u})-D \Delta \phi-\rho \mathbf{u}_{t} \cdot \nabla\left(\frac{\phi^{2}}{\alpha}\right) \\
& +(\operatorname{div} \widehat{\nabla \mathbf{u}}) \cdot \nabla\left(\frac{\eta}{\alpha \phi}\right)-\nabla \phi \cdot \nabla D+\operatorname{div}\left(\frac{\eta^{\prime}}{\alpha} \phi \widehat{\nabla \mathbf{u}} \nabla \phi\right) \\
& -\operatorname{div}\left(\frac{1}{\alpha} \rho \phi^{2} \nabla \mathbf{u u}\right)
\end{aligned}
$$

where $D$ is again the coefficient of diffusion as defined above. Taking the time derivative of $(2.3)_{2}$, multiplying by $\rho \phi / \alpha$ and rearranging terms

$$
\rho \frac{\phi}{\alpha} \phi \operatorname{div} \mathbf{u}_{t}=-\rho \frac{\phi}{\alpha}\left[\phi_{t t}+\phi_{t} \operatorname{div} \mathbf{u}+(\nabla \phi \cdot \mathbf{u})_{t}\right] .
$$

Remark. Naturally, we assume all functions together with their derivatives are continuous and so $(\operatorname{div} \mathbf{u})_{t}=\operatorname{div}\left(\mathbf{u}_{t}\right)$, etc.

Now taking the lapacian of $(2.3)_{2}$ and multiplying by $\eta / \alpha$,

$$
\frac{1}{\alpha} \eta \phi \operatorname{div}(\Delta \mathbf{u})=-\frac{\eta}{\alpha}\left[\Delta \phi_{t}+(\Delta \phi) \operatorname{div} \mathbf{u}+\Delta(\nabla \phi \cdot \mathbf{u})\right] .
$$

Finally, we substitute $(2.3)_{2},(2.6)$ and (2.7) into (2.5) to arrive at

$$
\begin{gathered}
\phi_{t}-D \Delta \phi+\rho \frac{\phi}{\alpha} \phi_{t t}-\frac{\eta}{\alpha} \Delta \phi_{t}=-\rho \frac{\phi}{\alpha} \phi_{t} \operatorname{div} \mathbf{u}-\rho \frac{\phi}{\alpha}(\nabla \phi \cdot \mathbf{u})_{t}+\frac{\eta}{\alpha}(\Delta \phi) \operatorname{div} \mathbf{u} \\
+\frac{\eta}{\alpha} \Delta(\nabla \phi \cdot \mathbf{u})+\rho \mathbf{u}_{t} \cdot \nabla\left(\frac{\phi^{2}}{\alpha}\right)-\operatorname{div}(\widehat{\nabla \mathbf{u}}) \cdot \nabla\left(\frac{\eta}{\alpha} \phi\right) \\
+\nabla \phi \cdot \nabla D-\operatorname{div}\left(\frac{\eta^{\prime}}{\alpha} \phi \widehat{\nabla \mathbf{u}} \nabla \phi-\frac{1}{\alpha} \rho \phi^{2} \nabla \mathbf{u u}\right) .
\end{gathered}
$$

If we now assume that $\mathbf{u}$, derivatives of $\mathbf{u}$ and derivatives $\phi$ are small, the right-hand side of (2.8) can be replaced by zero and hence

$$
\phi_{t}-D \Delta \phi+\rho \frac{\phi}{\alpha} \phi_{t t}-\frac{\eta}{\alpha} \Delta \phi_{t}=0
$$

Notice the first two terms are the classical diffusion equation. The third term is associated with inertia and the fourth term arises from the viscosity. The above equation is similar to one derived by Aifantis [5]. Without the inertia term equation (2.9) occurs often in the literature. Here we need only to assume that derivatives of $\mathbf{u}$ and derivatives of $\phi$ are small (cf. Williams [1]). In this case if $D$ and $\eta / \alpha$ are positive, which is a reasonable assumption (cf. Williams [1]), then the equation is well-behaved (see Ting [18], Barenblatt, Kochina and Zheltov [19], and Chen and Gurtin [20] for discussions of its properties).

III. Uniqueness in an unsaturated region. We will now examine the problem of uniqueness in an unsaturated body. Recall that the homogeneous medium is not completely filled with fluid (i.e., there may be air pockets) and hence the volume fraction is not constant with respect to time or space. The equations under consideration are (2.3). By a solution of (2.3) we mean functions: $\mathbf{u}: \mathscr{B} \times[0, T] \rightarrow \mathbf{R}^{3}$ and $\phi: \mathscr{B} \times[0, T] \rightarrow\left[0, \phi_{0}\right]$ 
that satisfy (2.3) and the following initial and boundary conditions:

$$
\begin{array}{ll}
\mathbf{u}(\mathbf{x}, 0)=\mathbf{u}_{0}(\mathbf{x}) & \text { for all } \mathbf{x} \in \mathscr{B}, \\
\phi(\mathbf{x}, 0)=\phi_{0}(\mathbf{x}) & \text { for all } \mathbf{x} \in \mathscr{B}, \\
\mathbf{u}(\mathbf{x}, t)=\mathbf{U}(\mathbf{x}, t) & \text { for all } \mathbf{x} \in \partial \mathscr{B} .
\end{array}
$$

It is not reasonable to specify the amount of fluid leaving the solid, that is, $\phi$ cannot be specified where $\mathbf{u} \cdot \mathbf{n}>0$. It is, however, reasonable to specify the amount of fluid flowing into the body. At such points $\mathbf{u} \cdot \mathbf{n}<0$, and here we specify $\phi(\mathbf{x}, t)=\Phi(\mathbf{x}, t)$.

We will assume that $\alpha, \beta$ and $\eta$ are $C^{1}$ functions and that the viscosity is nonnegative. It is now possible to state the following uniqueness theorem.

THEOREM 1. If $\eta>0$, then for every $T>0$ there is at most one classical solution of (2.3) satisfying (3.1) on $[0, T]$.

Proof. Suppose $\left(\phi_{1}, \mathbf{u}_{1}\right)$ and $\left(\phi_{2}, \mathbf{u}_{2}\right)$ are two solutions of (2.3) satisfying (3.1). Subtracting the two equations and letting $\mathbf{u}=\mathbf{u}_{1}-\mathbf{u}_{2}, \phi=\phi_{1}-\phi_{2}$ we see that, on rearrangement of terms

$$
\begin{gathered}
\rho \phi_{1} \mathbf{u}_{t}+\rho \phi\left(\mathbf{u}_{2}\right)_{t}+\rho \phi_{1} \nabla \mathbf{u} \mathbf{u}_{1}+\rho \phi_{1} \nabla \mathbf{u}_{2} \mathbf{u}+\rho \phi \nabla \mathbf{u}_{2} \mathbf{u}_{2} \\
=\operatorname{div}\left(\left(\eta\left(\phi_{1}\right)-\eta\left(\phi_{2}\right)\right) \widehat{\nabla \mathbf{u}_{1}}\right)+\operatorname{div}\left(\eta\left(\phi_{2}\right) \widehat{\nabla \mathbf{u}}\right)-\alpha\left(\phi_{2}\right) \mathbf{u} \\
-\left(\alpha\left(\phi_{1}\right)-\alpha\left(\phi_{2}\right)\right) \mathbf{u}_{1}-\nabla\left(\beta\left(\phi_{1}\right)-\beta\left(\phi_{2}\right)\right), \\
\phi_{t}=-\operatorname{div}\left(\phi \mathbf{u}_{1}\right)-\operatorname{div}\left(\phi_{2} \mathbf{u}\right),
\end{gathered}
$$

with $\mathbf{u}(\mathbf{x}, 0)=0, \phi(\mathbf{x}, 0)=0$ for all $\mathbf{x} \in \mathscr{B} ; \mathbf{u}(\mathbf{x}, t)=0$ for all $\mathbf{x} \in \partial \mathscr{B}$ and $\phi(\mathbf{x}, t)=0$ for all $\mathbf{x} \in \partial \mathscr{B}_{1}$ where $\partial \mathscr{B}_{1}(t)=\left\{\mathbf{x}: \mathbf{u}_{i}(\mathbf{x}, t) \cdot \mathbf{n}<0, i=1,2\right\}$. We will show that $\mathbf{u}(\mathbf{x}, t)$ and $\phi(\mathbf{x}, t)$ are zero for all $\mathbf{x} \in \mathscr{B}$ and $t \in[0, T]$. Multiplying (3.2) by $\phi_{1} \phi$, integrating over $\Re$, rewriting the left-hand side and applying the divergence theorem we obtain

$$
\frac{d}{d t} \int_{\mathscr{B}} \phi_{1} \frac{\phi^{2}}{2}=-\int_{\mathscr{B}} \phi_{1} \phi^{2} \operatorname{div} \mathbf{u}_{1}-\int_{\mathscr{B}} \phi_{1} \phi \nabla \phi_{2} \cdot \mathbf{u}-\int_{\mathscr{B}} \phi_{1} \phi_{2} \phi \operatorname{div} \mathbf{u}+N P,
$$

where $N P=-\int_{\partial \mathscr{B}}\left(\phi^{2} / 2\right) \phi_{1} \mathbf{u}_{1} \cdot \mathbf{n}$. From before we see that $\phi$ cannot be specified where $\mathbf{u}_{1} \cdot \mathbf{n}>0$. However at such points $N P<0$. Recall also that $\phi=0$ when $\mathbf{u}_{1} \cdot \mathbf{n}<0$, hence $N P=0$. Thus the last term, $N P$, is nonpositive.

Now we take the inner product of $(3.2)_{1}$ with $\mathbf{u}$. Rearranging terms, integrating over $\mathscr{B}$ and applying the divergence theorem to the appropriate terms we arrive at

$$
\begin{aligned}
\frac{d}{d t} \int_{\mathscr{B}} \rho \phi_{1} \frac{\|\mathbf{u}\|^{2}}{2} & =-\int_{\mathscr{B}}\left[\rho \phi\left(\left(\mathbf{u}_{2}\right)_{t}+\nabla \mathbf{u}_{2} \mathbf{u}_{2}\right)+\left(\alpha\left(\phi_{1}\right)-\alpha\left(\phi_{2}\right)\right) \mathbf{u}_{1}\right] \cdot \mathbf{u} \\
& -\int_{\mathscr{B}} \rho \phi_{1} \nabla \mathbf{u}_{2} \mathbf{u} \cdot \mathbf{u}-\int_{\mathscr{B}}\left(\eta\left(\phi_{1}\right)-\eta\left(\phi_{2}\right)\right) \widehat{\nabla \mathbf{u}_{1}} \cdot \widehat{\nabla \mathbf{u}} \\
& -\int_{\mathscr{B}} \eta\left(\phi_{2}\right)\|\widehat{\nabla} \mathbf{u}\|^{2}-\int_{\mathscr{B}} \alpha\left(\phi_{2}\right)\|\mathbf{u}\|^{2}+\int_{\mathscr{B}}\left(\beta\left(\phi_{1}\right)-\beta\left(\phi_{2}\right)\right) \operatorname{div} \mathbf{u},
\end{aligned}
$$

where we have used the boundary condition $\mathbf{u}(\mathbf{x}, t)=0$ for $\mathbf{x} \in \partial \mathscr{B}$.

Now the second integral on the right hand side of (3.4) is bounded by $\int_{\mathscr{B}} \phi_{1} \rho\left|\lambda_{m}\right|\|\mathbf{u}\|^{2}$ where $\lambda_{m}$ is the minimum eigenvalue of $\widehat{\nabla \mathbf{u}_{2}}$. Recall that $\alpha, \beta, \eta \in C^{1}\left[0, \phi_{0}\right]$ and so by 
applying the Mean Value Theorem to the appropriate terms and using the following arithmetic-geometric mean inequality on the first and third integral on the right-hand side

$$
\mathbf{y} \cdot \mathbf{z} \leqslant \frac{\varepsilon^{2}}{2}\|\mathbf{y}\|^{2}+\frac{1}{2 \varepsilon^{2}}\|\mathbf{z}\|^{2}
$$

(3.4) becomes the following inequality

$$
\begin{aligned}
\frac{d}{d t} \int_{\mathscr{B}} \rho \phi_{1} \frac{\|\mathbf{u}\|^{2}}{2} & \leqslant \int_{\mathscr{B}}\left[\frac{a^{2}}{2} \rho^{2}\|\mathbf{w}\|^{2}+\frac{1}{2 b^{2}} \| \eta^{\prime}\left(\xi_{3}\right) \widehat{\nabla \mathbf{u}_{1} \|^{2}}\right] \phi^{2} \\
& +\int_{\mathscr{B}}\left[\frac{1}{2 a^{2}}+\rho\left|\lambda_{m}\right| \phi_{1}-\alpha\left(\phi_{2}\right)\right]\|\mathbf{u}\|^{2} \\
& -\int_{\mathscr{B}}\left[\eta\left(\phi_{2}\right)-\frac{b^{2}}{2}\right] \| \widehat{\nabla \mathbf{u} \|^{2}} \\
& +\int_{\mathscr{B}} \beta^{\prime}\left(\xi_{2}\right) \phi \operatorname{div} \mathbf{u},
\end{aligned}
$$

where

$$
\mathbf{w}=\left(\mathbf{u}_{2}\right)_{t}+\nabla \mathbf{u}_{2} \mathbf{u}_{2}+\frac{1}{\rho} \alpha^{\prime}\left(\xi_{1}\right) \mathbf{u}_{1},
$$

$\xi_{1}, \xi_{2}, \xi_{3} \in\left(0, \phi_{0}\right)$ and $a^{2}$ and $b^{2}$ are arbitrary positive numbers.

REMARK. From Sec. II, the prime denotes the derivative with respect to $\phi$.

Again, using (3.5) on the second integral on the right-hand side of (3.3), dividing (3.6) by $\rho$ and adding these results, we arrive at

$$
\begin{aligned}
& \frac{d}{d t} \int_{\mathscr{B}} \phi_{1}\left[\frac{\|\mathbf{u}\|^{2}}{2}+\frac{\phi^{2}}{2}\right] \leqslant \int_{\mathscr{B}}\left[\frac{a^{2}}{2} \rho\|\mathbf{w}\|^{2}+\frac{1}{2 \rho b^{2}}\left\|\eta^{\prime}\left(\xi_{3}\right) \widehat{\nabla \mathbf{u}}_{1}\right\|^{2}+\frac{c^{2}}{2} \phi_{1}^{2}\left\|\nabla \phi_{2}\right\|^{2}-\phi_{1} \operatorname{div} \mathbf{u}_{1}\right] \phi^{2} \\
& +\int_{\mathscr{B}}\left[\frac{1}{2 \rho a^{2}}+\left|\lambda_{m}\right| \phi_{1}-\frac{\alpha\left(\phi_{2}\right)}{\rho}+\frac{1}{2 c^{2}}\right]\|\mathrm{u}\|^{2} \\
& -\int_{\mathscr{B}} \frac{1}{\rho}\left[\eta\left(\phi_{2}\right)-\frac{b^{2}}{2}\right] \| \widehat{\nabla \mathbf{u} \|^{2}} \\
& +\int_{\mathscr{B}} \phi\left[\frac{\beta^{\prime}\left(\xi_{2}\right)}{\rho}-\phi_{1} \phi_{2}\right] \operatorname{div} \mathbf{u},
\end{aligned}
$$

where $c^{2}$ is an arbitrary positive number.

Applying (3.5) to the last integral in (3.8) and using the fact that $(\operatorname{div} \mathbf{u})^{2} \leqslant 9\|\widehat{\nabla u}\|^{2}$ we have

$$
\int_{\mathscr{B}} \phi\left[\frac{\beta^{\prime}\left(\xi_{2}\right)}{\rho}-\phi_{1} \phi_{2}\right] \operatorname{div} \mathbf{u} \leqslant \int_{\mathscr{B}} \frac{1}{2 d^{2}}\left[\frac{\beta^{\prime}\left(\xi_{2}\right)}{\rho}-\phi_{1} \phi_{2}\right]^{2} \phi^{2}+\int_{\mathscr{B}} 9 \frac{d^{2}}{2}\|\widehat{\nabla} \mathbf{u}\|^{2},
$$

where $d^{2}$ is an arbitrary positive number. We choose $b$ and $d$ such that

$$
\frac{1}{\rho} \eta\left(\phi_{2}\right)-\frac{1}{\rho} \frac{b^{2}}{2}-9 \frac{d^{2}}{2}>0
$$


and fix $a$ and $c$. Now let

$$
\begin{aligned}
\kappa_{1}=\sup _{\mathbf{x} \in \mathscr{B}}\left[\frac { 1 } { \phi _ { 1 } } \left\{\frac{a^{2}}{2} \rho\|\mathbf{w}\|^{2}+\frac{1}{2 \rho b^{2}} \| \eta^{\prime}\left(\xi_{3}\right) \widehat{\nabla \mathbf{u}_{1}\left\|^{2}+\frac{c^{2}}{2} \phi_{1}^{2}\right\| \nabla \phi_{2} \|^{2}-\phi_{1} \operatorname{div} \mathbf{u}_{1}}\right.\right. \\
\left.\left.+\frac{1}{2 d^{2}}\left[\frac{\beta^{\prime}\left(\xi_{2}\right)}{\rho}-\phi_{1} \phi_{2}\right]^{2}\right\}\right]
\end{aligned}
$$

and

$$
\kappa_{2}=\sup _{\mathbf{x} \in \mathscr{B}}\left[\frac{1}{\phi_{1}}\left\{\frac{1}{2 \rho a^{2}}+\left|\lambda_{m}\right| \phi_{1}-\frac{\alpha\left(\phi_{2}\right)}{\rho}+\frac{1}{2 c^{2}}\right\}\right] .
$$

Setting $E(t)=\int_{\mathscr{B}} \phi_{1}\left(\|\mathbf{u}\|^{2} / 2+\phi^{2} / 2\right)$ and $\kappa=2 \sup _{t \in[0, T]}\left(\kappa_{1}, \kappa_{2}\right)$ we obtain $d E / d t \leqslant \kappa E$ so that

$$
E(t) \leqslant E(0) e^{\kappa t} \equiv 0 .
$$

Therefore $E(t) \equiv 0$ for all $t \in[0, T]$ and $\mathbf{u}(\mathbf{x}, t) \equiv 0$ and $\phi(\mathbf{x}, t) \equiv 0$. Hence $\mathbf{u}_{1}(\mathbf{x}, t) \equiv$ $\mathbf{u}_{2}(\mathbf{x}, t)$ and $\phi_{1}(\mathbf{x}, t) \equiv \phi_{2}(\mathbf{x}, t)$.

Notice that a crucial part of the above proof is the requirement that the viscosity $(\eta)$ is positive. Uniqueness can also be established even if the viscosity is zero.

THEOREM 2. Let $\eta=0$ but let $\beta$ be a strictly monotonically increasing function of $\phi$. Then for every $\mathrm{T}>0$ there is at most one classical solution of (2.3) satisfying (3.1) on $[0, T]$.

Proof. Assuming $\eta \equiv 0$, equation (3.6) becomes

$$
\begin{aligned}
\frac{d}{d t} \int_{\mathscr{B}} \rho \phi_{1} \frac{\|\mathbf{u}\|^{2}}{2} \leqslant & \int_{\mathscr{B}} \frac{a^{2}}{2} \rho^{2}\|\mathbf{w}\|^{2} \phi^{2} \\
& +\int_{\mathscr{B}}\left[\frac{1}{2 a^{2}}+\rho\left|\lambda_{m}\right| \phi_{1}-\alpha\left(\phi_{2}\right)\right]\|\mathbf{u}\|^{2}+\int_{\mathscr{B}} \beta^{\prime}\left(\xi_{2}\right) \phi \operatorname{div} \mathbf{u},
\end{aligned}
$$

where $\mathbf{w}$ is defined in (3.7).

We rewrite Eq. $(3.2)_{2}$ as

$$
\phi_{t}+\nabla \phi \cdot \mathbf{u}_{1}+\phi \operatorname{div} \mathbf{u}_{1}+\nabla \phi_{2} \cdot \mathbf{u}+\phi_{2} \operatorname{div} \mathbf{u}=0 .
$$

Multiply this by $\phi$ and rearrange terms to obtain

$$
\phi \operatorname{div} \mathbf{u}=-\frac{1}{\phi_{2}}\left(\left(\phi^{2} / 2\right)_{\mathbf{u}_{1}}^{\cdot}+\phi \nabla \phi_{2} \cdot \mathbf{u}+\phi^{2} \operatorname{div} \mathbf{u}_{1}\right),
$$

where ()$_{\mathbf{u}_{1}}^{\cdot}$ denotes the material derivative with respect to $\mathbf{u}_{1}$ (i.e., $\left.(f)_{\mathbf{u}_{1}}^{\cdot}=f_{t}+\nabla f \cdot \mathbf{u}_{1}\right)$. Then

$$
\int_{\mathscr{B}} \beta^{\prime}\left(\xi_{2}\right) \phi \operatorname{div} \mathbf{u}=-\int_{\mathscr{B}} \frac{\beta^{\prime}\left(\xi_{2}\right)}{\phi_{2}}\left(\phi^{2} / 2\right)_{\mathbf{u}_{1}}^{\cdot}-\int_{\mathscr{B}} \frac{\beta^{\prime}\left(\xi_{2}\right)}{\phi_{2}} \nabla \phi_{2} \cdot \phi \mathbf{u}-\int_{\mathscr{B}} \frac{\beta^{\prime}\left(\xi_{2}\right)}{\phi_{2}} \phi^{2} \operatorname{div} \mathbf{u}_{1} .
$$

Following Graffi [21] we see that if

$$
f=\frac{\beta^{\prime}\left(\xi_{2}\right)}{\phi_{1} \phi_{2}} \frac{\phi^{2}}{2}
$$


then we have that

$$
\begin{aligned}
\frac{d}{d t} \int_{\mathscr{B}} \phi_{1} f & =-\int_{\partial \mathscr{B}} f \phi_{1} \mathbf{u}_{1} \cdot \mathbf{n}+\int_{\mathscr{B}} \phi_{1}(f)_{\mathbf{u}_{1}}^{\cdot} \\
& =-\int_{\partial \mathscr{B}} \frac{\beta^{\prime}\left(\xi_{2}\right)}{\phi_{2}} \frac{\phi^{2}}{2} \mathbf{u}_{1} \cdot \mathbf{n}+\int_{\mathscr{B}} \phi_{1}\left[\left(\frac{\beta^{\prime}\left(\xi_{2}\right)}{\phi_{1} \phi_{2}}\right)_{\mathbf{u}_{1}}^{\cdot} \frac{\phi^{2}}{2}+\frac{\beta^{\prime}\left(\xi_{2}\right)}{\phi_{1} \phi_{2}}\left(\frac{\phi^{2}}{2}\right)_{\mathbf{u}_{1}}^{\cdot}\right] .
\end{aligned}
$$

The last integral of equation (3.9) then reads

$$
\begin{aligned}
\int_{\mathscr{B}} \beta^{\prime}\left(\xi_{2}\right) \phi \operatorname{div} \mathbf{u}= & -\int_{\mathscr{B}} \frac{\beta^{\prime}\left(\xi_{2}\right)}{\phi_{2}} \nabla \phi_{2} \cdot \phi \mathbf{u}-\int_{\mathscr{B}} \frac{\beta^{\prime}\left(\xi_{2}\right)}{\phi_{2}} \phi^{2} \operatorname{div} \mathbf{u}_{1}-\frac{d}{d t} \int_{\mathscr{B}} \frac{\beta^{\prime}\left(\xi_{2}\right)}{\phi_{2}} \frac{\phi^{2}}{2} \\
& -\int_{\partial \mathscr{B}} \frac{\beta^{\prime}\left(\xi_{2}\right)}{\phi_{2}} \frac{\phi^{2}}{2} \mathbf{u}_{1} \cdot \mathbf{n}+\int_{\mathscr{G}} \phi_{1}\left(\frac{\phi^{2}}{2}\right)\left(\frac{\beta^{\prime}\left(\xi_{2}\right)}{\phi_{1} \phi_{2}}\right)_{\mathbf{u}_{1}}^{\cdot}
\end{aligned}
$$

and equation (3.9) becomes

$$
\begin{aligned}
\frac{d}{d t} \int_{\mathscr{B}}\left[\rho \phi_{1} \frac{\|\mathbf{u}\|^{2}}{2}+\frac{\beta^{\prime}\left(\xi_{2}\right)}{\phi_{2}} \frac{\phi^{2}}{2}\right] \leqslant & \int_{\mathscr{B}}\left[\frac{a^{2}}{2} \rho^{2}\|\mathbf{w}\|^{2}-\frac{\beta^{\prime}\left(\xi_{2}\right)}{\phi_{2}} \operatorname{div} \mathbf{u}_{1}+\frac{\phi_{1}}{2}\left(\frac{\beta^{\prime}\left(\xi_{2}\right)}{\phi_{1} \phi_{2}}\right)_{\mathbf{u}_{1}}\right]^{2} \\
& +\int_{\mathscr{G}}\left[\frac{1}{2 a^{2}}+\rho\left|\lambda_{m}\right| \phi_{1}-\alpha\left(\phi_{2}\right)\right]\|\mathbf{u}\|^{2} \\
& -\int_{\mathscr{G} B} \frac{\beta^{\prime}\left(\xi_{2}\right)}{\phi_{2}} \nabla \phi_{2} \cdot \phi \mathbf{u}-\int_{\partial \mathscr{B}} \frac{\beta^{\prime}\left(\xi_{2}\right)}{\phi_{2}} \frac{\phi^{2}}{2} \mathbf{u}_{1} \cdot \mathbf{n} .
\end{aligned}
$$

Applying (3.5) to the third integral on the right-hand side (with $\varepsilon^{2}=1 / 2$ ), fixing $a$ and again noting that

$$
-\int_{\partial 囚} \frac{\beta^{\prime}\left(\xi_{2}\right)}{\phi_{2}} \frac{\phi^{2}}{2} \mathbf{u}_{1} \cdot n \leqslant 0
$$

(recall that $\beta$ is monotonically increasing, and hence that $\beta^{\prime}\left(\xi_{2}\right)$ is positive) we see that

$$
d E / d t \leqslant \kappa E,
$$

where

$$
\begin{aligned}
E(t)= & \int_{\mathscr{B}}\left(\rho \phi_{1} \frac{\|\mathbf{u}\|^{2}}{2}+\frac{\beta^{\prime}\left(\xi_{2}\right)}{\phi_{2}} \frac{\phi^{2}}{2}\right), \\
\kappa_{3}= & \sup _{\mathbf{x} \in \mathscr{B}}\left[\frac { \phi _ { 2 } } { \beta ^ { \prime } ( \xi _ { 2 } ) } \left(\frac{a^{2}}{2} \rho^{2}\|\mathbf{w}\|^{2}-\frac{\beta^{\prime}\left(\xi_{2}\right)}{\phi_{2}} \operatorname{div} \mathbf{u}_{1}\right.\right. \\
& \left.\left.+\frac{\phi_{1}}{2}\left(\frac{\beta^{\prime}\left(\xi_{2}\right)}{\phi_{1} \phi_{2}}\right)_{\mathbf{u}_{1}}^{\cdot}+\left(\frac{\beta^{\prime}\left(\xi_{2}\right)}{\phi_{2}}\right)^{2} \frac{\left\|\nabla \phi_{2}\right\|^{2}}{4}\right)\right], \\
\kappa_{4}= & \sup _{\mathbf{x} \in \mathscr{B}}\left[\frac{1}{\rho \phi_{1}}\left(\frac{1}{2 a^{2}}+\rho\left|\lambda_{m}\right| \phi_{1}-\alpha\left(\phi_{2}\right)+1\right)\right]
\end{aligned}
$$

and $\kappa=2 \sup _{t \in[0, T]}\left(\kappa_{3}, \kappa_{4}\right)$. As before, $E(t) \leqslant E(0) e^{\kappa t} \equiv 0$. Therefore $E(t) \equiv 0$ and $\mathbf{u}_{1}(\mathbf{x}, t) \equiv \mathbf{u}_{2}(\mathbf{x}, t)$ and $\phi_{1}(\mathbf{x}, t) \equiv \phi_{2}(\mathbf{x}, t)$. 
IV. Uniqueness in a totally saturated region. In this section we will prove classical uniqueness for various saturated regions. We consider three different situations, allowing the porous medium to be:

(1) inhomogeneous and time-dependent;

(2) inhomogeneous and time-independent;

(3) homogeneous and time-independent.

Remark 1. In the first case $\phi=\phi(\mathbf{x}, t)$. Here the pores will be allowed to vary with respect to time and space. This differs from being in an unsaturated region in that the pores are still completely filled with fluid, hence the pressure term is not necessarily zero as in an unsaturated body. We assume that, for each $\mathbf{x}$, the time derivative of $\phi(\mathbf{x}, t)$ is very small compared to the total volume of the region.

Remark 2. In the second situation $\phi=\phi(\mathbf{x})$ is specified. When working with models describing fluid flow it is common to assume that the pores are circular cylinders. In this case we assume that $\phi$ changes with respect to $\mathbf{x}$ and hence that the pore size can vary at each $\mathbf{x}$.

Remark 3. In the third case $\phi \equiv \phi_{0}$. The pores are now circular cylinders which do not change with respect to space or time and they are completely filled with fluid. See the remark at the end of the stability results for a statement of results in the weak sense.

In each situation the pores will be filled with fluid. In case one we are generalizing the theory to allow that the medium may be compressible and hence change porosity, still disallowing gross motion. We must also allow the pressure to vary with $\phi$.

Although the third case is a special case of the first two, it is of particular interest because of the similarity of these equations to the Navier-Stokes equations. We will show that even in the case of small or zero viscosity (high Reynolds numbers) the equations may still be well behaved if the drag coefficient is sufficiently large.

Consider the first case. Here the pores may change with time but will always be assumed to be filled with fluid. Notice that the volume fraction $(\phi)$ will change with time and on physical grounds it is reasonable to assume that the pressure $(p)$ may depend on $\phi$. Equations (2.1) will be used. Recall that the capillary effect $(\beta)$ is dependent on the volume fraction. Let $\tilde{\beta}=\beta+p$. Then $\tilde{\beta}=\tilde{\beta}(\phi)$ and we can now invoke the results proved for unsaturated regions, Theorem 1 when $\eta \neq 0$ and Theorem 2 when $\eta=0$ (see Sec. III).

We will now consider the second case. Here Eqs. (2.1) reduce to

$$
\begin{gathered}
\rho \phi \mathbf{u}_{t}+\rho \phi \nabla \mathbf{u u}=-\nabla p+\operatorname{div}(\eta(\phi) \widehat{\nabla \mathbf{u}})-\alpha(\phi) \mathbf{u}-\nabla \beta(\phi), \\
\operatorname{div}(\phi \mathbf{u})=0 .
\end{gathered}
$$

By a solution of (4.1) we mean functions $u$ : $\mathscr{B} \times[0, T] \rightarrow \mathbf{R}^{3}$ and $p: \mathscr{B} \times[0, T] \rightarrow[0, \infty)$ which satisfy (4.1) with

$$
\begin{array}{cl}
\mathbf{u}(\mathbf{x}, 0)=\mathbf{u}_{0}(\mathbf{x}) & \text { for all } \mathbf{x} \in \mathscr{B}, \\
\mathbf{u}(\mathbf{x}, t)=\mathbf{U}(\mathbf{x}, t) & \text { for all } \mathbf{x} \in \partial \mathscr{B} .
\end{array}
$$

Notice that $\phi(\mathbf{x})$ is a preassigned function. Since the porous medium is always saturated and incompressible, $\phi$ will be the same for all time. 
THEOREM 3. For every $T>0$ there is at most one classical solution of (4.1) satisfying the initial and boundary conditions (4.2) on $[0, T] .^{4}$

Proof. Let $\left(p_{1}, \mathbf{u}_{1}, \phi\right)$ and $\left(p_{2}, \mathbf{u}_{2}, \phi\right)$ be two solutions of (4.1) satisfying (4.2). Then $\mathbf{u}=\mathbf{u}_{1}-\mathbf{u}_{2}$ and $p=p_{1}-p_{2}$ must satisfy

$$
\begin{gathered}
\rho \phi \mathbf{u}_{t}+\rho \phi \nabla \mathbf{u} \mathbf{u}_{1}+\rho \phi \nabla \mathbf{u}_{2} \mathbf{u}=-\nabla p+\operatorname{div}(\eta(\phi) \widehat{\nabla \mathbf{u}})-\alpha(\phi) \mathbf{u}, \\
\operatorname{div}(\phi \mathbf{u})=0 .
\end{gathered}
$$

Taking the inner product of $(4.3)$, with $\phi \mathbf{u}$, integrating over $\mathscr{B}$, applying the divergence theorem and rearranging terms we obtain

$$
\begin{aligned}
\frac{d}{d t} \int_{\mathscr{B}} \rho \phi^{2} \frac{\|\mathbf{u}\|^{2}}{2}= & \int_{\mathscr{B}} \rho \operatorname{div}\left(\phi^{2} \mathbf{u}_{1}\right) \frac{\|\mathbf{u}\|^{2}}{2}-\int_{\mathscr{B}} \rho \phi^{2} \nabla \mathbf{u}_{2} \mathbf{u} \cdot \mathbf{u} \\
& -\int_{\mathscr{B}} \eta(\phi) \widehat{\nabla \mathbf{u}} \cdot \nabla(\phi \mathbf{u})-\int_{\mathscr{B}} \alpha(\phi) \phi\|\mathbf{u}\|^{2},
\end{aligned}
$$

where we have used the boundary condition $\mathbf{u}(\mathbf{x}, t)=0$ for $\mathbf{x} \in \partial \Re$ and the equation $\operatorname{div}(\phi \mathbf{u})=0$. Now

$$
\widehat{\nabla \mathbf{u}} \cdot \nabla(\phi \mathbf{u})=\widehat{\nabla \mathbf{u}} \cdot[\mathbf{u} \otimes \nabla \phi+\phi \nabla \mathbf{u}]=\mathbf{u} \cdot \widehat{\nabla \mathbf{u}} \nabla \phi+\phi\|\widehat{\nabla \mathbf{u}}\|^{2} .
$$

Substituting the above equation into (4.4), letting $\lambda_{m}$ be the minimum eigenvalue of $\widehat{\nabla \mathbf{u}_{2}}$ and applying (3.5) to the term $-\eta \mathbf{u} \cdot \widehat{\nabla \mathbf{u}} \nabla \phi$ we see that

$$
\begin{aligned}
\frac{d}{d t} \int_{\mathfrak{B}} \rho \phi^{2} \frac{\|\mathbf{u}\|^{2}}{2} \leqslant & \int_{\mathscr{B}}\left[\frac{1}{2} \rho \operatorname{div}\left(\phi^{2} \mathbf{u}_{1}\right)+\rho \phi^{2}\left|\lambda_{m}\right|-\alpha(\phi) \phi+\frac{\eta(\phi)^{2}}{2 a^{2}}\right]\|\mathbf{u}\|^{2} \\
& -\int_{\mathfrak{B}}\left[\eta(\phi) \phi-\frac{\|\nabla \phi\|^{2}}{2} a^{2}\right] \| \widehat{\nabla \mathbf{u} \|^{2},}
\end{aligned}
$$

where $a$ is arbitrary. But now let us choose $a$ such that

$$
\eta(\phi) \phi-\|\nabla \phi\|^{2} a^{2} / 2>0 .
$$

Letting

$$
\kappa=\sup _{\substack{\mathbf{x} \in \mathscr{G} \\ t \in[0, T]}}\left[\frac{2}{\rho \phi^{2}}\left(\frac{\rho}{2} \operatorname{div}\left(\phi^{2} \mathbf{u}_{1}\right)+\rho \phi^{2}\left|\lambda_{m}\right|-\alpha(\phi) \phi+\frac{\eta(\phi)^{2}}{2 a^{2}}\right)\right]
$$

and

$$
E(t)=\int_{\mathscr{B}} \rho \phi^{2} \frac{\|\mathbf{u}\|^{2}}{2},
$$

we have $d E / d t \leqslant \kappa E$ so that $E(t) \leqslant E(0) e^{\kappa t} \equiv 0$. Therefore $\mathbf{u}_{1}(\mathbf{x}, t)=\mathbf{u}_{2}(\mathbf{x}, t)$. Setting $\mathbf{u}=0$ in (4.3) yields $\nabla p=0$. Hence $p$ is unique up to an arbitrary function of time.

Remark. The proof of uniqueness is valid even with viscosity $(\eta)$ zero.

Let us now turn to the question of uniqueness in the case of a saturated, homogeneous medium that is not changing with time. The equations under consideration are (2.2).

\footnotetext{
${ }^{4} p$ will be unique up to a function of time.
} 
Again, notice the similarity of these to the incompressible Navier-Stokes equations.

By a solution of (2.2) we mean functions $u$ : $\mathscr{B} \times[0, T] \rightarrow \mathbf{R}^{3}$ and $p: \mathscr{B} \times[0, T] \rightarrow[0, \infty)$ which satisfy (2.2) and the initial and boundary conditions given in (4.2).

THEOREM 4. For every $T>0$ there is at most one classical solution of (2.2) satisfying (4.2) for all $t \in[0, T] .^{5}$

The proof of Theorem 3 applies here with $\phi=\phi_{0}$. Without the drag term, (2.2) are the Navier-Stokes equations and the standard classical proofs of uniqueness can be applied here also (cf. Joseph [22], Serrin [23]).

V. Stability in a totally saturated region. To examine the effects of viscosity and drag in classical stability we will consider the equations

$$
\begin{gathered}
\rho \phi_{0} \overline{\mathbf{u}}_{t}^{-}+\rho \phi_{0} \bar{\nabla} \overline{\mathbf{u}} \overline{\mathbf{u}}=-\bar{\nabla} \bar{p}+\eta_{0} \overline{\operatorname{div}}(\bar{\nabla} \overline{\mathbf{u}})-\alpha_{0} \overline{\mathbf{u}}, \\
\overline{\operatorname{div}} \overline{\mathbf{u}}=0
\end{gathered}
$$

which satisfy $\overline{\mathbf{u}}(\overline{\mathbf{x}}, 0)=\overline{\mathbf{u}}_{0}(\overline{\mathbf{x}})$ for all $x \in \Re$ and $\overline{\mathbf{u}}(\overline{\mathbf{x}}, t)=\overline{\mathbf{U}}(\overline{\mathbf{x}}, t)$ for all $\mathbf{x} \in \partial \Re$.

Let us begin by making the equations dimensionless. Choose a characteristic length $D$ and a characteristic velocity $V$, and let $\mathbf{u}=\overline{\mathbf{u}} / V, p=\bar{p} / \rho \phi_{0} V^{2}, t=\bar{t} V / D, x=\overline{\mathbf{x}} / D$, $\nabla=D \bar{\nabla}, \operatorname{div}=D \overline{\operatorname{div}}$, and $\partial / \partial t=D \partial / V \partial \bar{t}$. Hence, Eqs. (5.1) become

$$
\begin{gathered}
\mathbf{u}_{t}+\nabla \mathbf{u u}=-\nabla p+\frac{1}{R} \Delta \mathbf{u}-Q \mathbf{u}, \\
\operatorname{div} \mathbf{u}=0 .
\end{gathered}
$$

where $R=\rho V D \phi_{0} / \eta_{0}$ is the Reynolds number and $Q:=\alpha_{0} D / \rho V \phi_{0}$ is a dimensionless drag coefficient. Notice that $Q$ is positive and bounded above. Suppose we have two solutions $\left(\mathbf{u}_{1}, p_{1}\right)$ and $\left(\mathbf{u}_{2}, p_{2}\right)$ of (5.2) satisfying $\mathbf{u}_{1}(\mathbf{x}, t)=\mathbf{u}_{2}(\mathbf{x}, t)$ for all $\mathbf{x} \in \partial \mathscr{B}$. Then $\mathbf{u}=\mathbf{u}_{1}-\mathbf{u}_{2}$ and $p=p_{1}-p_{2}$ must satisfy

$$
\begin{aligned}
\mathbf{u}_{t}+\nabla \mathbf{u u}_{1}+\nabla \mathbf{u}_{2} \mathbf{u} & =-\nabla p+\frac{1}{R} \Delta \mathbf{u}-Q \mathbf{u}, \\
\operatorname{div} \mathbf{u} & =0
\end{aligned}
$$

with $\mathbf{u}(\mathbf{x}, t)=0$ for all $\mathbf{x} \in \partial \mathscr{B}$. Take the inner product of (5.3), with $\mathbf{u}$, integrate over $\Re$, and apply the divergence theorem. Upon rearrangement of terms, we arrive at

$$
\frac{d}{d t} \int_{\mathfrak{B}} \frac{\|\mathbf{u}\|^{2}}{2}=-\int_{\mathscr{B}} \nabla \mathbf{u}_{2} \mathbf{u} \cdot \mathbf{u}-\int_{\mathscr{B}} \frac{1}{R}\|\nabla \mathbf{u}\|^{2}-\int_{\mathscr{B}} Q\|\mathbf{u}\|^{2} .
$$

Now let $V=\{\mathbf{v}: \mathbf{v}=0$ on $\partial \mathscr{B}$ and $\operatorname{div} \mathbf{v}=0\}$ and

$$
r(t)=\max _{\mathbf{v} \in V} \frac{-\int_{\mathfrak{B}}\left(Q 1+\nabla \mathbf{u}_{2}\right) \mathbf{v} \cdot \mathbf{v}}{\int_{\mathfrak{B}}\|\nabla \mathbf{v}\|^{2}} .
$$

\footnotetext{
${ }^{5} \mathbf{u}$ is unique on $[0, T]$ and $p$ is unique up to a function of time.
} 
Then $r(t)$ is bounded above on $[0, T]$. Recall that $Q$ is bounded above and that $\nabla \mathbf{u}_{2} \mathbf{u} \cdot \mathbf{u} \leqslant\left|\lambda_{m}\right|\|\mathbf{u}\|^{2}$ where $\lambda_{m}$ is the minimum eigenvalue of $\widehat{\nabla \mathbf{u}}_{2}$. Also

$$
\int_{\mathscr{B}}\|\mathbf{u}\|^{2} / \int_{\mathscr{B}}\|\nabla \mathbf{u}\|^{2} \leqslant 1 / \Lambda
$$

where $\Lambda$ is the Poincare constant. ${ }^{6}$

Remark. It is easy to show that $\lambda_{m} \leqslant 0$. Recall that $0=\operatorname{div} \mathbf{u}_{2}=\operatorname{tr} \nabla \mathbf{u}_{2}=\operatorname{tr} \widehat{\nabla \mathbf{u}_{2}}$ so that at least one of the eigenvalues of $\widehat{\nabla} \mathbf{u}_{2}$ is negative. Note also that $\lambda_{m}$ is a function of time, hence $r$ is a function of time also.

Letting $E(t)=\int_{\mathscr{D}}\|\mathbf{u}\|^{2}$ we can now formulate the following stability result:

THEOREM 5. Let $r(t)$ be defined by (5.5) and suppose that one of the following conditions holds on $[0, T]$ :

(1) $Q \geqslant\left|\lambda_{m}\right|$;

(2) $1 / R \geqslant\left|\lambda_{m}\right| / \Lambda$; or

(3) $1 / R>r(t)$.

Then $\mathbf{u}$ is stable on $[0, T] \cdot{ }^{7}$ If, in addition,

(4) $1 / R>r_{M}=\sup _{t>0} r(t)$ or

(5) $Q \geqslant\left|\lambda_{m}\right|$ for all $t$.

Then $E(t)$ is globally and monotonically stable. ${ }^{8}$ Conversely, if $1 / R<r(0)$ then there exists a $\mathbf{u}_{0}(x) \in V$ such that $E(0)$ is initially unstable.

Proof. Rewrite (5.4) as

$$
\frac{d E}{d t}=-2 \int_{\mathscr{B}}\left(Q 1+\nabla \mathbf{u}_{2}\right) \mathbf{u} \cdot \mathbf{u}-2 \int_{\mathfrak{B}} \frac{1}{R}\|\nabla \mathbf{u}\|^{2} .
$$

Suppose that (1), (2), or (3) holds. Then it suffices to show that $d E / d t<-\kappa E$ where $\kappa>0$ on $[0, T]$, for then

$$
E(t) \leqslant E(0) \exp \left[-\int_{0}^{t} \kappa(\tau) d \tau\right]
$$

for all $t \in[0, T]$.

Case 1. If $Q \geqslant\left|\lambda_{m}\right|$ then $r(t) \leqslant 0$. Hence

$$
\frac{d E}{d t} \leqslant-\frac{2}{R} \int_{\mathscr{B}}\|\nabla \mathbf{u}\|^{2} \leqslant-\frac{2}{R} \Lambda E(t)
$$

and we set $\kappa=2 \Lambda / R$.

Case 2. If $1 / R \geqslant\left|\lambda_{m}\right| / \Lambda$ then

and we set $\kappa=2 Q$.

$$
\begin{aligned}
\frac{d E}{d t} & =-2 Q E-\frac{2}{R} \int_{\mathscr{B}}\|\nabla \mathbf{u}\|^{2}-2 \int_{\mathscr{B}} \nabla \mathbf{u}_{2} \mathbf{u} \cdot \mathbf{u} \\
& \leqslant-2 Q E-2\left(\frac{\Lambda}{R}-\left|\lambda_{m}\right|\right) E \leqslant-2 Q E,
\end{aligned}
$$

\footnotetext{
${ }^{6}$ Assuming $B$ is a regular region.

${ }^{7}$ Stable in the sense that two solutions initially close in the sense of $L^{2}(\mathscr{B})$ will remain close on $[0, T]$.

${ }^{8}$ In fact, all solutions tend to the zero solution exponentially.
} 
Case 3. $1 / R>r(t)$. Then

$$
\begin{aligned}
\frac{d E}{d t} & =-2 \int_{\mathscr{B}}\left(Q 1+\nabla \mathbf{u}_{2}\right) \mathbf{u} \cdot \mathbf{u}-\frac{2}{R} \int_{\mathscr{B}}\|\nabla \mathbf{u}\|^{2} \\
& \leqslant-2\left(\frac{1}{R}-r(t)\right) \int_{\mathscr{B}}\|\nabla u\|^{2} \leqslant-2\left(\frac{1}{R}-r(t)\right) \Lambda E,
\end{aligned}
$$

and we set $\kappa=2(1 / R-r(t)) \Lambda$.

Remark. Here the technique of Joseph [22] was used. Now, however, $r(t)$ contains an extra term, namely $Q$, which will have the effect of making $r(t)$ smaller as $Q$ gets larger.

To show global and monotonic stability, it suffices to show $\kappa>0$ for all time.

Case 4. $1 / R>r_{M}=\sup _{t>0} r(t)$. Then

$$
\frac{d E}{d t} \leqslant-2\left(\frac{1}{R}-r_{M}\right) \Lambda E
$$

and if we set $\kappa=2\left(1 / R-r_{M}\right) \Lambda$, then $\kappa>0$ for all time.

Case 5. If $Q \geqslant\left|\lambda_{m}\right|$ for all $t$ then $r(t) \leqslant 0$ for all $t$ and

$$
\frac{d E}{d t} \leqslant-\frac{2}{R} \Lambda E \text {. }
$$

Set $\kappa=2 \Lambda / R$.

Remark. Recall that $Q$ depends on $\alpha_{0}$ and so if the drag is sufficiently large, this is possible.

On the other hand, $\mathbf{u}$ will be initially unstable if there exists a $\kappa>0$ such that $d E /\left.d t\right|_{t=0} \geqslant \kappa E(0)$. Suppose at $t=0, \mathbf{w}=\mathbf{u}_{0}(x)$ maximizes $r(t)$. Then

$$
\begin{aligned}
\left.\frac{d E}{d t}\right|_{t=0} & =2\left\{\frac{-\int_{\mathscr{B}}\left(Q 1+\nabla \mathbf{u}_{2}\right) \mathbf{w} \cdot \mathbf{w}}{\int_{\mathscr{B}}\|\nabla \mathbf{w}\|^{2}}-\frac{1}{R}\right\} \int_{\mathscr{B}}\|\nabla \mathbf{w}\|^{2} \\
& =-2\left(\frac{1}{R}-r(0)\right) \int_{\mathscr{B}}\|\nabla \mathbf{w}\|^{2} \geqslant \kappa E,
\end{aligned}
$$

where $1 / R<r(0)$. Here $\kappa=2(r(0)-1 / R) \Lambda$,

When showing instability we can immediately rule out the case $r(0) \leqslant 0$, since $1 / R$ (the Reynolds number) is always nonnegative. Recall that $r(0) \leqslant 0$ is possible only if the drag is sufficiently large $\left(Q>\left|\lambda_{m}(x, 0)\right|\right)$.

Remark. It is quite easy to apply the weak theory established for the Navier-Stokes system to this case. Let $\mathscr{B}$ be a bounded open set in $\mathbf{R}^{3}$ whose boundary is twice continuously differentiable, let $V$ denote the closure in $L^{2}(\Re)$ of $\{\mathbf{u} \in \mathscr{D}(\mathscr{B}): \operatorname{div} \mathbf{u}=0\}$ and let $H^{2}(\mathscr{B})=\left\{\mathbf{u}: \mathbf{u}, \nabla \mathbf{u}, \nabla(\nabla \mathbf{u}) \in L^{2}(\mathscr{B})\right\}$. Then one can easily establish the existence of a unique and stable solution in the space $L^{\infty}(0, T ; V) \cap L^{2}\left(0, T ; H^{2}(\Re)\right)$ for some $T$, by modifying the proof of Teman [24]. In particular, one must replace the bilinear form

$$
(A \mathbf{u}, \mathbf{v})=\int_{\mathscr{B}} \nabla \mathbf{u} \cdot \nabla \mathbf{v}
$$


by

$$
(A \mathbf{u}, \mathbf{v})=\int_{\mathscr{B}} \nabla \mathbf{u} \cdot \nabla \mathbf{v}+\frac{\alpha_{0}}{\eta_{0}} \int_{\mathscr{B}} \mathbf{u} \cdot \mathbf{v} .
$$

But it is easy to see that the modified $A$ is still coercive and hence that the results remain valid.

\section{REFERENCES}

[1] W. O. Williams, Constitutive equations for flow of an incompressible viscous fluid through a porous medium, Q. Appl. Math. 36 (1978), 255-267

[2] W. O. Williams, On the theory of mixtures, Arch. Rational Mech. Anal. 51 (1973), 239-260

[3] R. Sampaio and W. O. Williams, Thermodynamics of diffusing mixtures, J. Méchanique 18 (1979), 1945-1979

[4] K. A. Pericak-Spector and W. O. Williams, On work and constraints in mixtures, ZAMP (to appear)

[5] E. C. Aifantis, On the problem of diffusion in solids, Acta Mechanica 37 (1980), 265-296

[6] J. W. Nunziato and E. K. Walsh, On ideal multiphase mixtures with chemical reactions and diffusion, Arch. Rational Mech. Anal. 73 (1980), 285-311

[7] D. S. Drumheller, The theoretical treatment of a porous solid using a mixture theory, Int. J. Solids, Struct. 14 (1978), 441-456

[8] A. Bedford and D. S. Drumheller, A variational theory of immiscible mixtures, Arch. Rational Mech. Anal. 68 (1978), 37-51

[9] D. E. Kenyon, The theory of an incompressible solid-fluid mixture, Arch. Rational Mech. Anal. 62 (1976), $131-147$

[10] M. E. Gurtin and G. de la Penha, On the thermodynamics of mixtures; I. Mixtures of rigid heat conductors, Arch. Rational Mech. Anal. 36 (1970), 390-410

[11] M. L. Oliver, On balanced interactions in mixtures, Arch. Rational Mech. Anal. 49 (1972), 195-224

[12] K. A. Pericak-Spector, On flow through a porous medium, Ph. D. Thesis, Department of Mathematics, Carnegie-Mellon University (1980)

[13] H. C. Brinkman, A calculation of the viscous force exerted by a flowing fluid on a dense swarm of particles, Appl. Sci. Res. A1 (1947), 27-34

[14] H. C. Brinkman, On the permeability of media consisting of closely packed porous particles, Appl. Sci. Res. A1 (1947), 81-86

[15] H. C. Brinkman, Problems of fluid flow through swarms of particles and through macromolecules in solution, Research 2 (1949), 190-194

[16] R. B. Bird, W. E. Stewart and E. N. Lightfoot, Transport phenomena, Wiley, New York, 1960

[17] A. E. Scheidegger, The physics of flow through porous media, University of Toronto Press, Toronto, 1974

[18] T. W. Ting, Certain non-steady flows of second order fluids, Arch. Rational Mech. Anal. 14 (1963), 1-26

[19] G. I. Barenblatt, In P. Zheltov and I. N. Kochina, Basic concepts in the theory of seepage of homogeneous liquids in fissured rocks, J. Appl. Math. and Mechanics, PMM 24 (1960), 1286-1303 (transl. of Priklad Mat. Mech. 24 (1960), 852-864)

[20] P. J. Chen and M. E. Gurtin, On a theory of heat conduction involving two termperatures, Zeit. fur Angewandte Math. 19 (1968), 614-627

[21] D. Graffi, Il teorema di unicità nella dinamica dei fluidi compressible, JRMA 2 (1953), 99-106

[22] D. D. Joseph, Stability of fluid motions I, Springer-Verlag, Berlin, 1976

[23] J. Serrin, Mathematical principles of classical fluid mechanics, Handbuch der Physik VIII/1, Springer-Verlag, Berlin, 1959

[24] R. Teman, Navier-Stokes equations, North-Holland, New York, 1977 